\title{
Temporal limits to the detection of correlation in transpositionally symmetric dynamic dot textures
}

\author{
BILL JENKINS \\ University of Western Australia, Nedlands, Western Australia, Australia
}

\begin{abstract}
Adding a delay within spatially correlated point-pairs constituting a dynamic transpositionally symmetric texture has three distinct perceptual outcomes (the specific outcome dopending on the size of the delay): global pattern, coherent motion, and apparent randomness. The temporal limits for global pattern and coherent motion were measured for eight different values of the extent of transposition (.11 to .70 deg of visual angle) and for four different stim. ulus sizes $(3,5,7$, and 9 deg square), with texture density constant at the retina. Temporal limits for coherent motion varied inversely as a function of increasing spatial separation (.11 deg < $\Delta \mathrm{d}<.42 \mathrm{deg})$, but beyond this separation (.42 deg $<\Delta \mathrm{d}<.70 \mathrm{deg})$, the limit was invariant as a function of $\Delta d(\sim 60 \mathrm{msec}$ ). The temporal limit for global pattern was independent of the size of $\Delta \mathrm{d}$ and fell at $\sim 50 \mathrm{msec}$, exhibiting spatiotemporal characteristics different from those for coherent motion. On the basis of this difference, it is suggested that the human visual system processes the perceptual attributes global pattern and coherent motion independently, despite their common correlational basis. Further evidence to support this proposal is provided by the finding that variations in stimulus size have no effect on the temporal limit for global pattern and have differential effects on the temporal limit for coherent motion, depending on the size of $\Delta d$.
\end{abstract}

The use of random-dot textures, first described by Julesz (1960), has revealed a previously unexpected ability of the human visual system, that of detecting correlations between groups of points both binocularly (e.g., Julesz, 1960; Ross \& Hogben, 1974) and monocularly (e.g., Glass, 1969; Hobgen, Julesz, \& Ross, 1976). Investigations (in the monocular case, using bilateral, rotational, and transpositional symmetries) of the underlying mechanisms that mediate this correlative ability have manipulated both spatial and temporal characteristics of the elements that constitute the symmetric textures (e.g., Anstis, 1970; Barlow \& Reeves, 1979; Bell \& Lappin, 1979; Braddick, 1974; Glass \& Switkes, 1976; Hogben, Julesz, \& Ross, 1976; Jenkins, 1982a, 1982b; Lappin \& Bell, 1976; Morgan \& Ward, 1980).

Using bilaterally symmetric dot patterns, in which each point has a partner reflected across an axis that is the midpoint for all pairs in the field, Barlow and Reeves (1979) manipulated the relative position of symmetric points by "jittering" one dot of each bilateral pair about its exact symmetric position. Similarly, but using transpositionally symmetric dot patterns, in which a set of random dots is transposed

This research was supported by the Australian Research Grants Scheme through Grant A281159711 to the author. H. J. Jenkins is thanked for her invaluable research assistance. The author's mailing address is: Psychology Department, University of Western Australia, Nedlands, Western Australia 6009, Australia. uniformly along a particular orientation and superimposed on the set of dots in its original position, Glass and Switkes (1976) examined tolerance for the manipulation of relative position. They perturbed points in a random fashion along an arc subtending a range of angles about the orientation of the transposition, at a fixed separation from the original dot. Both these procedures demonstrated some tolerance for spatial manipulation, with transpositional symmetry allowing a greater degree of manipulation than bilateral symmetry before the breakdown in symmetry detection occurred. Breakdown in the detection of transpositional symmetry also occurs as the separation of the two patterns is increased (Jenkins, $1982 \mathrm{~b}$ ), revealing a linear spatial limitation to the detection of correlations within the patterns, a limitation similar to the disparity limits that exist for stereopsis.

In addition to these spatial limitations, the temporal tolerance of these correlational mechanisms has been investigated using dot textures (e.g., Bell \& Lappin, 1979; Braddick, 1974; Hogben, Julesz, \& Ross, 1976; Lappin \& Bell, 1976; Morgan \& Ward, 1980). The general finding is that tolerance for delayed correlated information is a rapidly decreasing function of an increase in the relative extent of transformation, whether the dot textures are transposed uniformly in one direction (e.g., Braddick, 1974; Lappin \& Bell, 1976; Morgan \& Ward, 1980) or rotated about a common center (Anstis, 1970; Bell \& Lappin, 1979). It also holds between different con- 
struction techniques; that is, this spatiotemporal interaction seems to result whether two discrete dot textures are used (e.g., Lappin \& Bell, 1976) or whether the correlated textures are built up dynamically by the sequential plotting of identical pointpair elements (e.g., Morgan \& Ward, 1980). In the latter case, the delay is not between two discrete dot textures, but is imposed within each point-pair element in the field, so that as a delay is added to individual point-pair elements, other points that constitute the stimulus stream can still be plotted within this interval.

Detection of correlation in the studies described above, with the exception of that by Hogben et al. (1976), utilized, as a basis for measuring the temporal tolerance, the perceptibility of coherent motion that results when delays are added to correlated textures. In the Hogben et al. study, the addition of delay between correlated elements that constituted a bilaterally symmetric dynamic dot texture resulted in the deterioration of global pattern to apparent randomness, without any intervening coherent motion. The detection of correlation in this case relied totally upon the presence of nonrandom global pattern. Uniformity of transposition may be a requisite for the perception of coherent motion.

An additional observation made independently by the author and by Morgan and Ward (1980) is that with dynamically constructed transpositionally symmetric textures, there are three quite distinct stages that occur when a uniform delay is imposed between the correlated points. First, as the delay is added, there is no apparent change in the stimulus texture, and the global pattern is still perceptible. Further delay results in a breakdown of the global pattern and the emergence of coherent motion. Additional delay results in a texture that is perceptually indistinguishable from an equally dense random-dot texture.

These observations suggest that the correlational mechanism can maintain, up to a point, the percept of global pattern despite interposed delay. Similarly, within limits, it can support the perceptibility of coherent motion.

A direct examination of the temporal limits to these two distinct correlational phenomena on the basis of completed studies is difficult, since there is no common stimulus type, correlational type, stimulus size, or density, etc. Therefore, it is the aim of this study to measure the temporal limits to these two phenomena and to determine whether they both conform to established spatiotemporal characteristics.

The two spatial parameters that will be examined using dynamic transpositionally symmetric textures (see Jenkins, 1982b, and Morgan \& Ward, 1980) are the extent of translation and stimulus size, with stimulus density held constant at the retina.
Experiment 1 measured the temporal limit for coherent motion as a function of extent of translation, $\Delta d$. Experiment 2 measured the temporal limit for global pattern as a function of the same set of values of $\Delta d$. Experiment 3 investigated both temporal limits as a function of stimulus size, for the same set of $\Delta d$ values. The use of transpositionally symmetric dot textures throughout these experiments allowed the direct comparison of the limits to both phenom. ena, since both global pattern and coherent motion occur in these textures, depending upon the particular value of the delay, $\Delta t$, within the spatially correlated point-pairs.

\section{METHOD}

The measurements utilized dynamic dot textures plotted on a display oscilloscope under computer control (PDP-8/e). The subjects viewed the stimulus binocularly, at a viewing distance of $57.3 \mathrm{~cm}$. Stimulus luminance was held constant at $1.2 \mathrm{log}$ units above threshold, with the oscilloscope face luminance maintained at $1 \mathrm{~cd} / \mathrm{m}^{2}$ (Spectra Pritchard photometer, Model 1980A-PL, 1-deg aperture). The display oscilloscope was a HewlettPackard 1332A, equipped with an ultrashort P 24 phosphor. The intensity of a point plotted on this type of phosphor has a single peak with a maximum spectral energy emission at $510 \mathrm{~nm}$, and the point fades to $10 \%$ of its original brightness in $1.5 \mu$ sec.

The construction of dynamic textures involves the rapid sequential plotting of points on an oscilloscope face within a square $9 \times 9 \mathrm{~cm}$ region. This region was a $256 \times 256$ matrix, so that the location of any point was given by one of 256 possible $x$-values and one of $\mathbf{2 5 6}$ possible $y$-values.

Dynamic transpositionally symmetric textures were constructed by sequentially plotting independent horizontal point-pairs with the same $\Delta d$. The position of each horizontal point-pair was determined randomly: the $x$ - and $y$-values were selected independently of each other (original point) and then the original $x$-value was incremented by $\Delta d$, providing the $x$-coordinate for the transposed point. The $y$-coordinate for this point was the same as that of the original point. Both points, the original and the transposed, were then plotted together with a duration of $1.5 \mu s e c$ and with an interval of $100 \mu \mathrm{sec}$ between the plotting of successive independent point-pairs. It must be emphasized that the dynamic textures constitute a continuous train of independent point-pair elements, and not a sequence of textured "frames" in the cinematographic manner (e.g., Lappin \& Bell, 1976).

The construction described above applies to the case in which the original and transposed points are plotted within $5 \mu \mathrm{sec}$. In order to investigate temporal tolerance for correlated texture, it was necessary to impose a delay, $\Delta t$, between the correlated points constituting the texture. Delay was achieved in the following manner: once a point-pair had been selected, a delay $(\Delta t)$ between the original and transposed points was obtained by lagging the plotting of one of the two points with respect to the plotting of the other. To ensure that there was no uniform spatial direction of the delay, the lagged point could be randomly either the original or the transposed.

The selection and plotting of new point-pairs continued at the rate of one pair every $100 \mu \mathrm{sec}$, irrespective of the size of $\Delta t$, which could vary continuously through 0 to $190 \mathrm{msec}$. It was therefore possible to maintain a continuous stream of point-pairs of a constant density, both spatial and temporal, at the retina, whether or not the stream contained a delay.

To measure the extent of memory for global pattern and coherent motion, the method of adjustment was used. The subject manipulated $\Delta t$ until a continuously plotted, transpositionally 
symmetric texture had no apparent global pattern or no coherent motion, depending upon the experimental requirements. This adjustment of $\Delta t$ was achieved by the use of a set of hand-held buttons interfaced to the PDP-8/e computer, which enabled the subject to increment or decrement the current value of $\Delta t$ while the textures were being plotted continuously. When the required criterion had been reached, the subject pressed a third button, which produced a printout of this threshold value of $\Delta t$. Twenty such thresholds were obtained from each subject, providing a mean threshold value for each $\Delta d$ investigated. A fourth button that could be pressed at any time produced a standard comparison stimulus-an equally dense dynamic random-dot texture-that subjects were instructed to use in the case of coherent motion. This was provided because, once coherent motion had disappeared, the remaining texture was perceptually indistinguishable from an equally dense random-dot texture.

Eight values of $\Delta d$ were investigated: $.11, .17, .28, .35, .42, .49$, .60 , and $.70 \mathrm{deg}$. All these values were within the spatial limitation to the perfect detection of transpositional symmetry, as reported by Jenkins (1982b), and all values of $\Delta d$ produced a very strong global pattern with no delay within correlated pairs. At the beginning of each block of 20 trials, the experimenter selected the value of $\Delta \mathrm{d}$ randomly from the defined set. The subject initiated the stimulus stream, and the value of $\Delta t$ was then adjusted by the subject until the criterion had been reached. The starting value of $\Delta t$ was selected randomly on each trial, and the size of the adjustment increment was selected randomly on each trial from one of five possible values: $1,2,3,4$, or 5 msec. Three experienced subjects were used; all had normal or corrected-to-normal vision.

\section{EXPERIMENT 1}

The aim of Experiment 1 was to determine whether the memory for correlated information giving rise to coherent motion varied as a function of the extent of translation, $\Delta \mathrm{d}$.

Stimulus size was fixed at $7 \mathrm{deg}$ square of visual angle, and the subjects were instructed to adjust $\Delta t$ until the dynamic texture contained no impression of coherent motion and was perceptually indistinguishable from an equally dense, dynamic random-dot field.

\section{Results and Discussion}

The results of Experiment 1, illustrated in Figure 1, demonstrate the relationship between $\Delta d$ and $\Delta t$ for the perceptibility of coherent motion in transpositionally symmetric dynamic dot textures.

The following aspects of these results should be noted: (1) The general trend of the memory estimates for correlated information varies inversely as a function of $\Delta d$ (for .11 deg $\leqslant \Delta d<.42 \mathrm{deg}$ ), ranging from $\sim 170 \mathrm{msec}$ to $\sim 50 \mathrm{msec}$, respectively. (2) For $\Delta \mathrm{d}>.42 \mathrm{deg}$, there is no consistent variation in the memory estimates; they fall generally within the 5070-msec region.

These results indicate that, given the addition of a delay, the visual system is able to detect correlated information more efficiently if the spatial separation between the correlated textures is small (e.g., .11 deg of visual angle). Smaller $\Delta \mathrm{d}$ values could have been investigated, but within these dynamic textures, decreasing the separation below $\sim .11$ deg made pattern

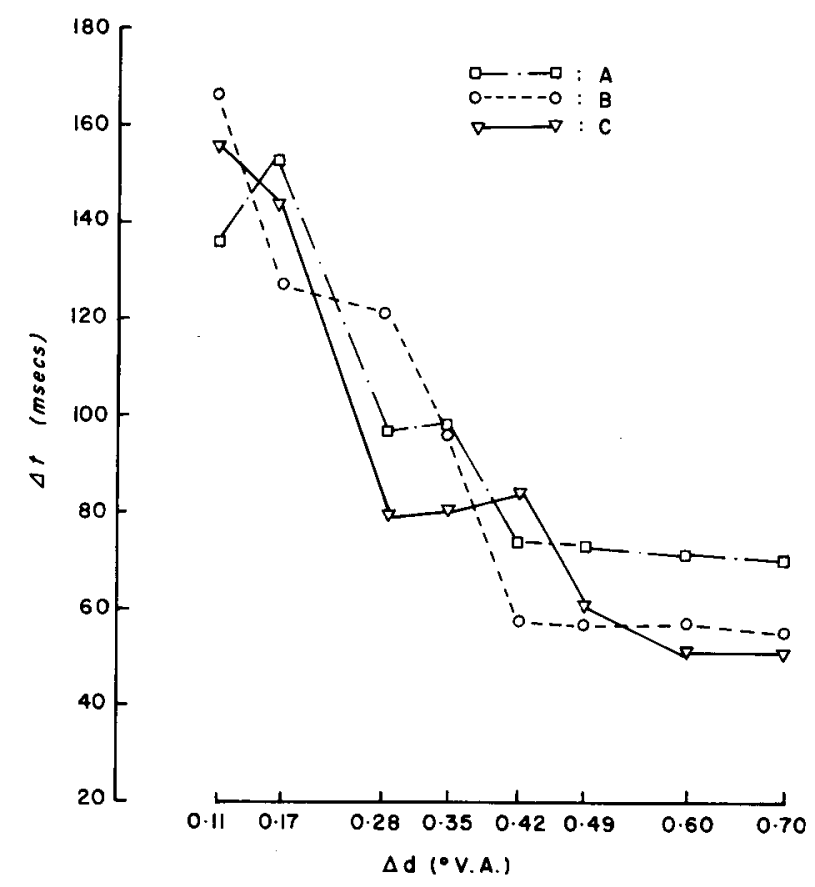

Figure 1. Results of Experiment 1. Tolerable delay (threshold $\Delta t)$ is plotted as a function of $\Delta d$ (extent of spatial displacement), for three subjects, using the criterion of "randomness."

resolution difficult. However, larger $\Delta d$ values $(\Delta d>$ $.42 \mathrm{deg}$ ), while providing an equally strong global pattern, demonstrated quite a different spatiotemporal interaction, with $\Delta d$ having no differential effect on memory for correlation. It seems that the visual system is less able to match the correlated textures as they are separated in time and space when $\Delta d$ $\leqslant .42 \mathrm{deg}$, but that, beyond this point, the matching ability is not affected by spatial separation (within the spatial boundaries for global-pattern perception in these textures-see Jenkins, 1982b).

This first characteristic is consistent with findings by Braddick (1974) and Lappin and Bell (1976) with respect to static dot textures, although a direct comparison of actual memory duration is impossible on several grounds.

The difference in the shape of the function for measured memory above and below $\Delta \mathrm{d}=.42 \mathrm{deg}$ could be the result of observers' adopting different criteria for the different values of $\Delta d$, but this is an unlikely possibility since there was an objective comparison stimulus available, that is, the equally dense random-dot texture, which all observers used frequently for all values of $\Delta d$. In addition, if the criterion had varied, then this would have been reflected in the distribution of measurements with $\Delta \mathrm{d}$; but the standard errors are small ( $\sim 2 \mathrm{msec}$ ) and approximately equal across different values of $\Delta d$, which indicates a high degree of consistency within and between $\Delta d$ values. 
The examination of the spatiotemporal interaction beyond $\Delta \mathrm{d}=.70 \mathrm{deg}$ would be useful to determine whether the asymptote evident in Figure 1 for all observers is maintained. Unfortunately, increasing the spatial separation, $\Delta \mathrm{d}$, beyond this figure results in a weakening of the impression of global pattern at zero delay, making direct comparison between different $\Delta d$ values unsound.

Having established the extent of spatiotemporal interaction associated with the memory for correlated information (in transpositionally symmetric textures) that gives rise to coherent motion, Experiment 2 measures temporal limits for global pattern in identical textures.

\section{EXPERIMENT 2}

The aim of Experiment 2 was to measure the memory for correlated information giving rise to global pattern and to determine whether this memory varied as a function of $\Delta d$.

Stimulus size was again fixed at $7 \mathrm{deg}$ square of visual angle, with all conditions being identical to those of Experiment 1. However, in this experiment, the subjects were instructed to adjust $\Delta t$ until the dynamic texture contained no global pattern.

\section{Results and Discussion}

The results of Experiment 2, shown in Figure 2, demonstrate that the memory for global pattern has a duration of approximately $50 \mathrm{msec}$ and that it does not vary consistently as a function of $\Delta d$.

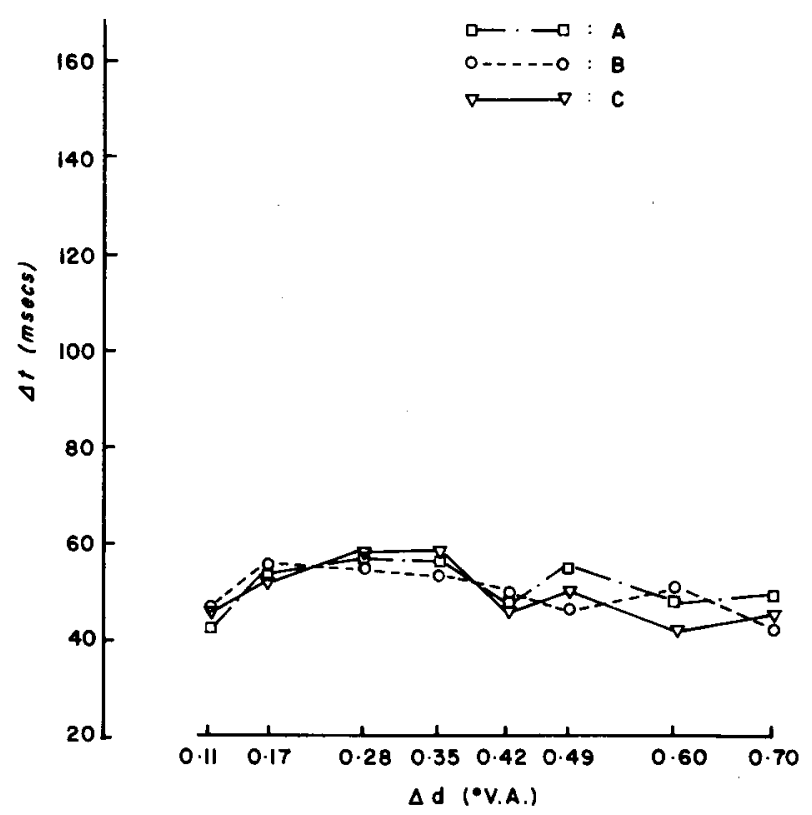

Figare 2. Reaults of Experiment 2. Tolerable delay is plotted a a function of $\Delta d$, for three subjectu, uaing the criterion "no global pattern."
These results indicate that the visual system is able to detect correlated information supporting global pattern independently of the spatial separation, indicating no spatiotemporal interaction within the measured limits.

This absence of spatiotemporal interaction contrasts strongly with the interaction demonstrated in the previous experiment for $\Delta \mathrm{d}<.42 \mathrm{deg}$. This difference in measured temporal tolerance for the two distinct perceptual outcomes of correlated textures with interposed delays, global pattern and coherent motion, suggests that the visual system may process each of these stimulus characteristics independently. In other words, there may be separate channels for processing pattern and motion.

This notion of independent channels, within the visual system, that process different stimulus dimensions and different values within a given dimension is not new (see Braddick, Campbell, \& Atkinson, 1978). In fact, evidence in support of this proposal of separate channels for pattern and motion has been provided by Tolhurst (1973) and by others on the basis, more specifically, of the investigation of correlative phenomena (e.g., Anstis, 1970; Lappin \& Bell, 1976; Morgan \& Ward, 1980). However, these latter investigators seem to emphasize the similarity of the two processes, in particular the importance of the spatial displacement between the two correlated textures. Experiments 1 and 2 in this investigation, however, illustrated differences, with the motion channel displaying the traditional spatiotemporal interaction (within limits) and the global-pattern channel demonstrating that its memory is constant and apparently indifferent to the spatial separation of the correlated textures.

Two questions that emerge as a result of these measurements are: (1) What factor or factors are responsible for these temporal limits and (2) are the same factors responsible for these limits in both channels?

In the motion channel, decreasing mean tolerable delay as a function of increasing spatial displacement suggests that one factor contributing to the memory limitation in this channel may be interference. As $\Delta \mathbf{d}$ is increased, with plotting rate held constant, the probability that uncorrelated noise points will fall between correlated points increases. Therefore, the limit may represent some critical value within the visual system, based on the signal-to-noise ratio.

However, in the pattern channel, the memory limit appears impervious to interference from uncorrelated points, since it does not change as the probability of interference increases, with increasing $\Delta d$. Therefore, it may be the case that the two channels have different bases for their temporal limits.

A test of this interference hypothesis as a basis for the temporal limit in both cases would be to vary the signal-to-noise ratio and to examine the subsequent 
effect on the measured memory. Varying the size of the stimulus texture, while keeping the plotting rate constant, results in a disproportionate increase in noise relative to signal. Increasing stimulus size results in an increase in the number of correlated pointpairs available to the visual system. The signal therefore increases linearly as a function of increasing stimulus size; however, noise increases at a greater rate. This results in a decrease in the signal-to-noise ratio as the stimulus size is increased. Therefore, if the temporal limits are based on interference, then as the stimulus size increases, the temporal limit should decrease. Experiment 3 examined the effect of stimulus size on the temporal limits for global pattern and coherent motion.

\section{EXPERIMENT 3}

The aim of Experiment 3 was to examine the effect of stimulus size on memory for global pattern and coherent motion.

To achieve this, Experiments 1 and 2 were repeated using three additional stimulus sizes, 3,5 , and $9 \mathrm{deg}$ square. Variation in stimulus size was obtained by keeping the plotting rate and viewing distance constant, and by masking the oscilloscope screen to the desired extent.

\section{Results and Discussion}

The effect of stimulus size is shown in Figures 3 and 4. First, Figure 3 demonstrates that changing the stimulus size has no qualitative effect on memory for global pattern or for coherent motion; the shapes of the respective functions remain unchanged. Quantitative comparisons are best made by examining Figure 4. The obvious constancy of temporal limitation

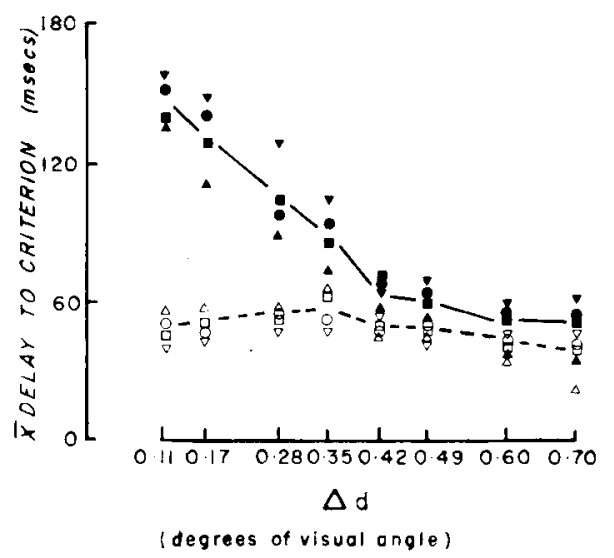

Figure 3. Results of Experiment 3. Mean delay to criterlon is plotted as a function of $\Delta d$ for both criteria ("randomness" = solid points; "no global pattern" = hollow points), for four stimulus sizes (3 deg square, vertical triangle; 5 deg square, square; 7 deg square, circle; 9 deg square, inverted triangle). The data are pooled across subjects. The Hnes join the means of the different stimulus sizes.

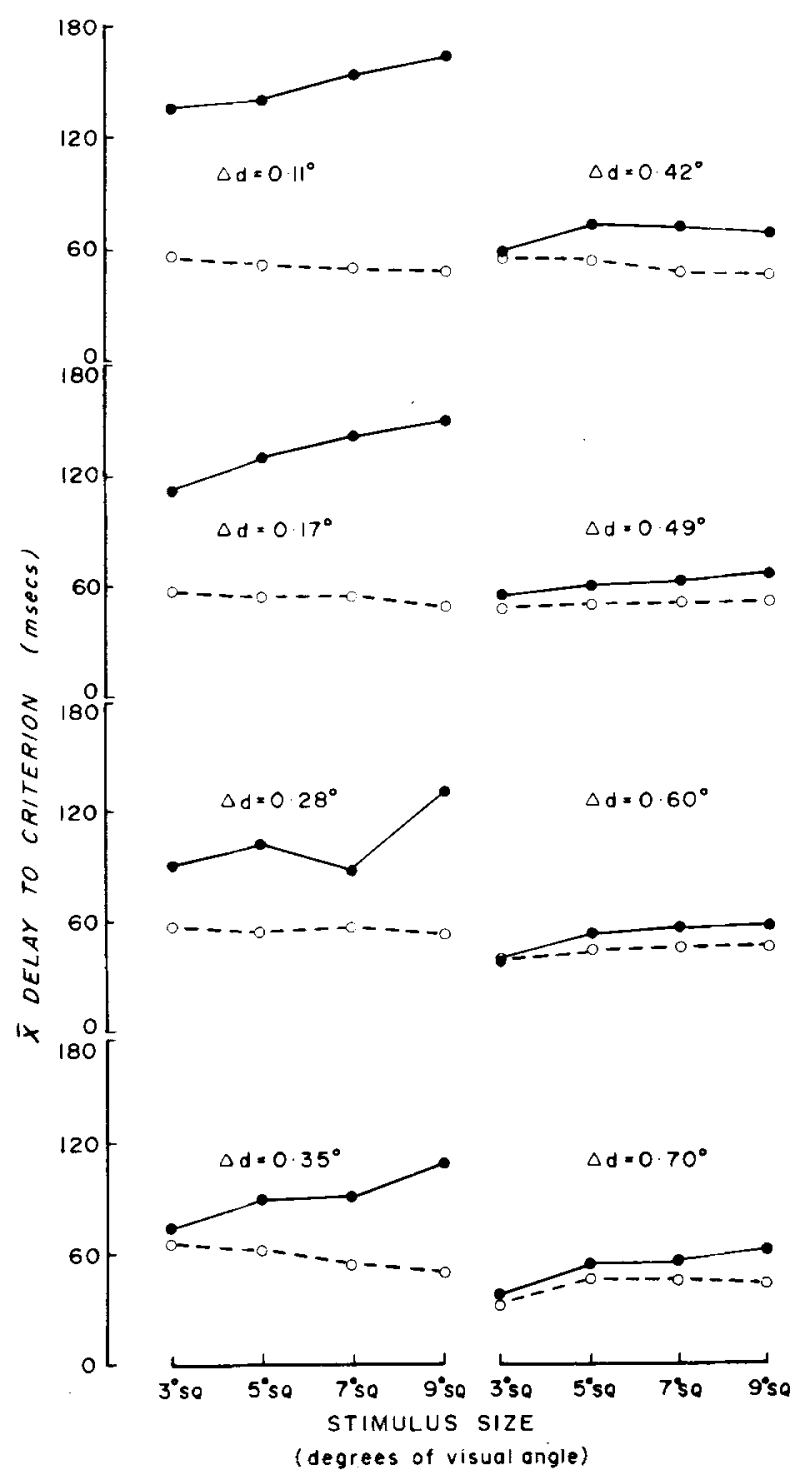

Figure 4. Results of Experiment 3. Mean delay to criterion is plotted as a function of stimulus size for individual values of $\Delta d$, for both criteria ("randomness" = solid points and line; "no global pattern" = hollow points and dahed line). The data are pooled across subjects. The diameter of the data polnts corresponds to the largest obtained standard error.

for global pattern provides a suitable baseline for both the qualitative and quantitative variations in memory for coherent motion, both as a function of $\Delta \mathrm{d}$ and of stimulus size.

There is no consistent effect of stimulus size on the temporal limit for global pattern, for any value of $\Delta d$. Tolerable delay for coherent motion, however, as a function of this factor, is more complex. Overall there is an effect of stimulus size $[F(3,6)=13.6$, $p<.05]$ and an effect of $\Delta d[F(7,14)=53.5, p<.05]$; however, there is also a significant interaction between stimulus size and $\Delta d[F(21,42)=2.06, p<.05]$, 
which can best be considered by comparing the values of $\Delta d<.42 \mathrm{deg}$ and those of $\Delta d>.42 \mathrm{deg}$ (see the left and right sides of Figure 4, respectively). For the former $\Delta \mathrm{d}$ values, increasing stimulus size results, generally, in an increase in tolerable delay; for the latter, however, there is no apparent trend.

The results of Experiment 3 add further evidence for the distinction between channels that process global pattern and coherent motion. Not only do these channels have different spatiotemporal characteristics, but these characteristics behave in different ways as a function of increasing stimulus size.

The interference hypothesis, at least in its simple form, as expressed above, is not consistent with these data. Increasing noise by increasing stimulus size does not result in the expected decline in temporal limit; in fact, for $\Delta d<.42 \mathrm{deg}$, tolerable delay in the coherent-motion channel increases with stimulus size, compared with no systematic variation at all for $\Delta d>.42$ deg. This suggests that, in addition to the distinction between channels for global pattern and coherent motion, a distinction must be made within this latter channel on the basis of the extent of spatial displacement.

If the limits of both channels were directly related to the correlational process, then increasing the number of signal elements should result in more effective correlation and, presumably, therefore, less susceptibility to noise. This hypothesis is consistent with the limit in the coherent-motion channel for $\Delta \mathrm{d}<.42 \mathrm{deg}$, since for these values of $\Delta d$, increasing the signal strength results in an increase in tolerable delay. However, this hypothesis does not account for the remaining findings. Increasing signal strength has no effect on memory for global pattern or for memory for coherent motion beyond $\Delta d=.42 \mathrm{deg}$.

However, several other factors covary with increasing stimulus size. As the size increases, with the intensity of points and the plotting rate held constant, the total intensity of the stimulus increases, although the average luminance per unit area remains constant. The total number of correlated elements also increases, although the density per unit area remains constant. A further factor is the heterogeneity of the retina: with an increase in size, more eccentric parts of the retina are stimulated and these differentiable areas may have different temporal responses. The effect of each of these factors is currently being investigated.

With no delay, the limit to the perceptibility of global pattern in transpositionally symmetric textures has been investigated by Jenkins (1982b), who found that the limit varied as a function of stimulus size, position on the retina, and frequency composition, but remained constant with variations in plotting rate, which suggests that the spatial limit may not be a function of interference (a hypothesis suggested by other investigators; e.g., Stevens, 1978) but may be due to some structural limitation within the correlational mechanism. By analogy from the spatial to the temporal domain, the apparent complexity of the spatiotemporal characteristics of the correlative mechanism demonstrated by Experiments 1, 2, and 3 suggests that the limitation may not be a function of simple interference or of correlational efficiency, but of the temporal response characteristics of likesensitive cell types constituting different channels in the human visual system.

\section{REFERENCES}

Ansts, S. M. Phi movement as a subtraction process. Vision Research, 1970, 10, 1411-1430.

Barlow, H. B., \& Reeves, B. C. The versatility and absolute efficiency of detecting mirror symmetry in random dot displays. Vision Research, 1979, 19, 783-793.

BELI, H. H., \& LAPPIN, J. S. The detection of rotation in randomdot patterns. Perception \& Psychophysics, 1979, 26, $415-417$.

Braddicx, O. A short-range process in apparent motion. Vision Research, 1974, 14, 519-527.

Braddick, O., Campeeld, F. W., \& Atringon, J. Channels in vision: Basic aspects. In R. Held, H. Leibowitz, \& H. L. Teuber (Eds.), Handbook of sensory physiology (Vol. 8): Perception. Heidelberg: Springer-Verlag, 1978.

Glass, L. Moiré effect from random dots. Nature, 1969, 223, 578-580.

Gla8s, L., \& Switkes, E. Pattern recognition in humans: Correlations which cannot be perceived. Perception, 1976, 5, 67-72.

Hoaben, J. H., Julesz, B., \& Ross, J. Short-term memory for symmetry. Vision Research, 1976, 16, 861-866.

Jenkins, B. Redundancy in the perception of bilaterally symmetric dot textures. Perception \& Psychophysics, 1982, 32, 171-177. (a)

JENKINs, B. Spatial limits to the detection of transpositional symmetry in dynamic dot textures. Journal of Experimental Psychology: Human Perception and Performance, 1982, in press. (b)

JuLEsz, B. Binocular depth perception of computer-generated patterns. Bell System Technical Journal, 1960, 39, 1125-1162.

LAPPIN, J. S., \& BELL, H. H. The detection of coherence in moving random-dot patterns. Vision Research, 1976, 16, 161-168.

Morone, M. J., \& WARD, R. Conditions for motion flow in dynamic visual noise. Vision Research, 1980, 20, $431-435$.

Ross, J., \& Hocken, J. H. Short-term memory in stereopsis. Vision Research, 1974, 14, 1195-1201.

Stevens, K. A. Computation of locally parallel structure. Biological Cybernetics, 1978, 29, 19-28.

TOLHUR8T, D. J. Separate channels for the analysis of shape and the movement of a moving visual stimulus. Joumal of Physiology, 1973, 231, 385-402.

(Manuscript réceived April 6, 1982; revision accepted October 25, 1982.) 